\title{
Numerical Simulations of Glide Dislocations in Persistent Slip Band
}

\author{
M. KOLÁ $\check{R}^{a, *}$, M. Beneš ${ }^{a}$, J. KRATOChVÍL ${ }^{b}$ AND P. PAUŠ ${ }^{a}$ \\ ${ }^{a}$ Department of Mathematics, Faculty of Nuclear Sciences and Physical Engineering, \\ Czech Technical University in Prague, Trojanova 13, Prague, Czech Republic \\ ${ }^{b}$ Department of Physics, Faculty of Civil Engineering, Czech Technical University in Prague, \\ Thákurova 7, Prague, Czech Republic
}

\begin{abstract}
For the purpose of estimation of possible inaccuracy in standard discrete dislocation dynamics simulations, we study the motion of interacting dislocations in two regimes: the standard stress control and the total strain control. For demonstration of the difference, we consider two dislocations of opposite signs, gliding in parallel slip planes in a channel of a persistent slip band. Exposed to the applied stress, the dislocations move, bow out, and form a dipole. We investigate the passing stress needed for the dislocations to escape each from other, considering the stress controlled regime and the total strain controlled regime. The motion is described by the mean curvature flow and treated by means of the direct (parametric) method. The results of numerical experiments indicate that the stress control and the total strain control provide upper and lower estimate of the passing stress, respectively, and that these two estimates differ by approximately $10 \%$.
\end{abstract}

DOI: 10.12693/APhysPolA.128.506

PACS: 02.70.Bf, 61.72.Lk, 02.30.Jr

\section{Model}

In standard discrete dislocation dynamics (DDD), it is assumed that the applied stress exerted on dislocation in the modeled crystal volume (see [1, 2]) is uniform; this regime is called stress controlled. In reality, the stress can be controlled only through boundary conditions applied to the material sample. However, the solution of the boundary value problem in DDD requires a rather complex discrete-continuum approach $[3,4]$. To estimate a possible inaccuracy in standard DDD, we employ another approach. Parallel to the stress control we evaluate a same DDD problem assuming that the sum of elastic and plastic strain is in the crystal volume uniform, the so-called total-strain controlled regime.

In the stress control, the elastic strain, coupled through the Hooke law to the stress, remains uniform (see [2]). Therefore, it cannot adjust to the generally nonuniform plastic strain produced by the dislocation glide (the compatibility of total strain in the channel is violated). Such artifical rigidity causes the stress level to be higher than in reality. In the total strain control, on the other hand, it is not required that the stress satisfies the stress equilibirum (only the equilibrium of the forces exerted on the dislocation lines are guaranteed by equation of their motion). Accordingly, the result applied stress is smaller than the correct one. The reality is between these two limits.

For estimation of a possible inaccuracy in standard DDD simulations, we focus on the interaction of two

* corresponding author; e-mail: kolarmir@fjfi.cvut.cz distinct dislocations moving in the opposite direction in two parallel slip planes exposed to the applied stress within the channel of the persistent slip band (PSB) evaluated for the two above described loading regimes. In our simulations, we consider the following geometrical settings (compare to $[3,4]$ ). Two initially straight dislocations $\Gamma^{(1)}$ and $\Gamma^{(2)}$ of the opposite signs with the Burgers vector $\boldsymbol{b}=(b, 0,0)$ and the ends fixed at the PSB walls are initially parallel to the $x$-axis of the $x, y$ and $z$ coordinate system. The dislocations glide in two slip planes of distance $h$ parallel to the $x z$-plane in the PSB channel of the width $d_{c}$. We consider the motion of the dislocations restricted to their slip planes. The minimum value of the distance $h$, which equals the maximum distance, at which the unlike screw dislocations can annihilate one another by cross-slip, determines the maximum stress achievable. In the present version of the simulation, the cross-slip is excluded. Their cross-slip and the critical annihilation distance $h$ were studied, e.g., in [5].

As the dislocations approach each other, their mutual attractive interaction becomes stronger, they are attracted speeding their motion. However, in their proximity the interaction becomes repulsive, which slows down their motion. Depending on the distance of the slip planes $h$ and on the exerted forces, the dislocations either stop at a dipole position or pass each other and continue to glide within the channel. Our goal is to investigate the applied stress needed for passing each other provided the distance of slip planes is given.

The dynamics of a planar dislocation can be approximately described by motion law known as the mean curvature flow (see [6-8])

$$
B v=T \kappa+F,
$$


where $B$ is the drag coefficient, $v$ is the normal velocity, $T$ is the line tension, $\kappa$ is the mean curvature and $F$ is the sum of all external forces acting on the dislocation. The self force generated by dislocation itself is approximated by the term $T \kappa$. For the line tension, the approximate formula $T=E^{(e)}\left(1-2 \nu+3 \cos ^{2}(\varphi)\right)$ is used (see [9]). Here $E^{(e)}$ is the dislocation edge energy, $\nu$ is the Poisson ratio and $\varphi$ is the angle between the tangent to the dislocation and the Burgers vector $\boldsymbol{b}$ with the magnitude $b$. The forcing term is expressed as $F=b \tau_{\text {tot }}$, where $\tau_{\text {tot }}$ represents the local resolved shear stress acting on the dislocation. In this article, we consider three following contributions to the resolved shear stress, i.e., the stress generated by the walls of the channel, the interaction stress, and the applied stress

$$
\tau_{\text {tot }}=\tau_{\text {wall }}+\tau_{\text {int }}+\tau_{\text {app }} .
$$

The structure of matured persistent slip bands consists of areas with low density of dislocations, i.e., the channel itself, where the dislocations in consideration glide, and of areas with high density of clustered edge dipolar loops, i.e., the channel walls. The channel walls cause a short range interaction, which is simulated as the elastic field of infinite edge dipoles. As the dislocation curve bows out under the effect of other exerted forces, the segments of the dislocation attracted by the wall are captured in the elastic potential wells of the dipoles. The resolved shear stress $\tau_{\text {wall }}$ exerted by the channel wall can be described by an approximate formula (see e.g. [6, 8]).

As the one gliding dislocation is influenced by another dislocation lying in a different parallel slip plane, one has to determine the tensor field $\tau_{\text {int }}=\left(\tau_{\text {int }}\right)_{i, j}$ describing the stress interaction. This problem was theoretically solved by Devincre in [1]. Generally, the force acting on the dislocation exposed to the stress field $\tau_{\text {int }}$ is given by the Peach-Koehler formula [10]. Since we consider a particular geometrical setting with the Burgers vector $\boldsymbol{b}$ parallel to the $x$-axis and the slip planes parallel to the plane $y=0$, it is possible (by direct calculation as in [8]) to determine the interaction force as $F_{\text {int }}=b \tau_{1,2}$ and thus to identify the interaction tensor with just one particular component $\tau_{1,2}$, which reduces the computational complexity.

The only external factor considered in our model is the stress applied on a crystal $\tau_{\text {app }}$. In this contribution, we investigate two simplified limit cases, whereas the reality is somewhere between these two limits. In the stress control regime, which is frequently used in discrete dislocation dynamics (for detailed analysis, see [4, 11]), we suppose the applied stress is uniform and constant in time, i.e., the resolved shear stress $\tau_{\text {app }}=$ const. The second limit case of the total strain control regime, we suppose a constant total shear strain rate, i.e., the uniform total shear strain $\varepsilon_{\text {tot }}(t)=\dot{\varepsilon} t$ with the time rate $\dot{\varepsilon}$, and we decompose it into the elastic part $\tau_{\text {app }} / \mu$ and the plastic part $\varrho b \int_{0}^{t} v(u, \tau) \mathrm{d} \tau$. Here $\mu$ is the shear modulus, $\varrho$ is the density of glide dislocations and $v$ is the normal velocity of the dislocation. The applied stress in the strain control regime is expressed as

$$
\tau_{\text {app }}(u, t)=\mu\left[\dot{\varepsilon} t-\varrho b \int_{0}^{t} v(u, \tau) \mathrm{d} \tau\right] .
$$

The visual difference between the dislocation shapes evolving in these two regimes can be seen in Fig. 1. In numerical experiments, the plastic relaxation $\varrho b \int_{0}^{t} v(u, \tau) \mathrm{d} \tau$ and slipped area are both approximated by means of areas of parallelograms, which are constructed on dual grid. Technical details can be found in [4].

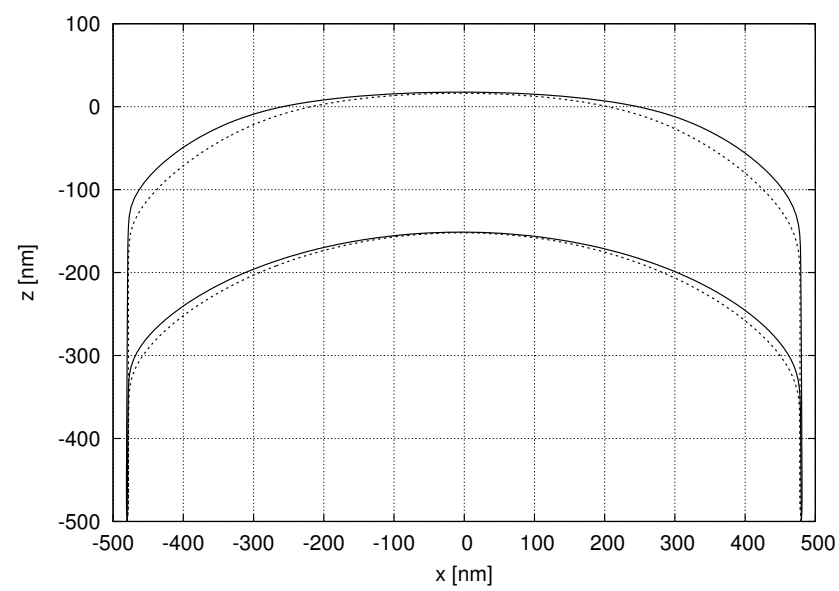

Fig. 1. Comparison of the stress and strain control regimes near the forming dipole position and before the forming dipole position. The dotted line represents the stress control regime with $\tau_{\text {app }}=44 \mathrm{MPa}$ depicted at the time levels $t=0.56 \mathrm{~ns}$ and $t=0.36 \mathrm{~ns}$, whereas the solid line represents the strain control regime at the time levels $t=1.16 \mathrm{~ns}$ and $t=0.89 \mathrm{~ns}$.

\section{Parametric method}

In this paper, the motion law (1.1) is treated by the direct method (also known as parametric or Lagrangian see [6-8]). The dislocation as the smooth time-dependent planar curve $\Gamma(t)=\left\{\operatorname{image}(\boldsymbol{X}(u, t)): u \in I_{u}\right\}$ is parametrized by means of the mapping $\boldsymbol{X}: I_{u} \times I_{t} \rightarrow \mathbb{R}^{2}$. Here $I_{u}=[0,1]$ is the fixed interval for the spatial parameter $u$ and $I_{t}=\left[0, T_{\max }\right]$ is the time interval.

Consequently, the geometric quantities of interest can be expressed by means of the mapping $\boldsymbol{X}$. We define the unit tangential vector $\boldsymbol{t}$ to the curve $\Gamma$ as $\boldsymbol{t}=\partial_{u} \boldsymbol{X} /\left|\partial_{u} \boldsymbol{X}\right|$. The outer unit normal vector $\boldsymbol{n}=$ $\partial_{u} \boldsymbol{X}^{\perp} /\left|\partial_{u} \boldsymbol{X}\right|$, where $\perp$ is the symbol of perpendicularity, is defined in such a way that $\boldsymbol{n} \cdot \boldsymbol{t}=0$ and $\operatorname{det}(\boldsymbol{n}, \boldsymbol{t})=1$ holds.

The normal velocity $v$ is the time derivative of the position vector $\boldsymbol{X}$ projected to the normal direction, i.e., $v=\partial_{t} \boldsymbol{X} \cdot \boldsymbol{n}$. Using the Frenet formulae, one can express the curvature as

$$
\kappa=\frac{\partial_{u u} \boldsymbol{X}}{\left|\partial_{u} \boldsymbol{X}\right|^{2}} \cdot \boldsymbol{n}=\frac{\partial_{u u} \boldsymbol{X}}{\left|\partial_{u} \boldsymbol{X}\right|^{2}} \cdot \frac{\partial_{u} \boldsymbol{X}^{\perp}}{\left|\partial_{u} \boldsymbol{X}\right|} .
$$

Then, $\Gamma$ evolves according to (1.1), provided $\boldsymbol{X}$ satisfies 
the following system of parametric equations:

$$
B \partial_{t} \boldsymbol{X}=T \frac{\partial_{u u} \boldsymbol{X}}{\left|\partial_{u} \boldsymbol{X}\right|^{2}}+F \frac{\partial_{u} \boldsymbol{X}^{\perp}}{\left|\partial_{u} \boldsymbol{X}\right|} .
$$

Since dislocations are investigated as open curves, Eqs. (2.1) are accompanied by the fixed-end boundary conditions, i.e., $\left.\boldsymbol{X}\right|_{u=0}=\boldsymbol{X}_{A},\left.\boldsymbol{X}\right|_{u=1}=\boldsymbol{X}_{B}$, and by the initial condition $\left.\boldsymbol{X}\right|_{t=0}=\boldsymbol{X}_{\text {ini }}(u)$ (a straight line in our case).

In numerical experiments using parametric Eqs. (2.1) the discretization points sometimes can accumulate in certain segments of the discretized curve. This unintended phenomenon occurs especially when the curve is exposed to a time and space variable force (as, e.g., in [12]). One possible way to overcome this problem is employing a tangential redistribution of the discretization points, i.e., adding a (possibly nonlocal) tangential velocity $\alpha$, which redistributes the discretization points along the curve without affecting its shape. This modification is extensively studied, e.g., in [13] and further developed in $[5,6,8]$. The redistribution increases the accuracy and the stability of the computational algorithm. For our computations, we use the curvature adjusted tangential redistribution as in [13] modified for open curves with fixed endpoints as in [8].

Then, the dynamics of two dislocations $\Gamma^{(1)}$ and $\Gamma^{(2)}$ in a PSB channel is governed by the system of equations

$$
\begin{gathered}
B \partial_{t} \boldsymbol{X}^{(1)}=T\left(\frac{\partial_{u u} \boldsymbol{X}^{(1)}}{\left|\partial_{u} \boldsymbol{X}^{(1)}\right|^{2}}+\alpha^{(1)} \frac{\partial_{u} \boldsymbol{X}^{(1)}}{\left|\partial_{u} \boldsymbol{X}^{(1)}\right|}\right) \\
+F^{(1)}\left(t, \boldsymbol{X}^{(1)}, \boldsymbol{X}^{(2)}\right) \frac{\partial_{u} \boldsymbol{X}^{(1) \perp}}{\left|\partial_{u} \boldsymbol{X}^{(1)}\right|}, \\
B \partial_{t} \boldsymbol{X}^{(2)}=T\left(\frac{\partial_{u u} \boldsymbol{X}^{(2)}}{\left|\partial_{u} \boldsymbol{X}^{(2)}\right|^{2}}+\alpha^{(2)} \frac{\partial_{u} \boldsymbol{X}^{(2)}}{\left|\partial_{u} \boldsymbol{X}^{(2)}\right|}\right) \\
+F^{(2)}\left(t, \boldsymbol{X}^{(1)}, \boldsymbol{X}^{(2)}\right) \frac{\partial_{u} \boldsymbol{X}^{(2) \perp}}{\left|\partial_{u} \boldsymbol{X}^{(2)}\right|},
\end{gathered}
$$

where the evaluation of the force terms $F^{(1)}$ and $F^{(2)}$ is described e.g. in [6] and the evaluation of the redistribution terms $\alpha^{(1)}$ and $\alpha^{(2)}$ is described e.g. in [8].

For discretization of Eq. (2.2), the finite difference method and the semi-implicit time stepping are used. The resulting linear systems are solved by matrix factorization at each time step. The parameters of the tangential redistribution can be found in [8].

\section{Computational results}

In this section we present the results of numerical simulations of two dislocations gliding in the opposite direction in a PSB channel. The numerical simulations were performed for copper with the parameters listed in Table.

As stated in Sect. 1, dislocations either approach the steady state and form a dipole, or pass and continue to glide. In Fig. 2, one can see the time evolution of passing dislocations in the strain controlled regime. The only external factor is the applied stress $\tau_{\text {app }}$. In the following, we determine how to measure the passing stress $\tau_{\text {pass }}$, i.e., the magnitude of the applied stress $\tau_{\text {app }}$, which suffices to break the steady state and force dislocations to glide.
TABLE

Parameters of the simulation.

\begin{tabular}{c|c}
\hline \hline Parameter & Value \\
\hline Burgers vector magnitude & $b=0.256 \mathrm{~nm}$ \\
Dislocation edge energy & $E^{(e)}=2.35 \mathrm{nN}$ \\
Drag coefficient & $B=1.0 \times 10^{-5} \mathrm{~Pa} \cdot \mathrm{s}$ \\
Plane distance & $h=50 \mathrm{~nm}$ \\
Channel width & $d_{c}=960 \mathrm{~nm}$ \\
Shear modulus & $\mu=42.1 \mathrm{GPa}$ \\
Poisson ratio & $\nu=0.43$ \\
Density of glide dislocations & $\varrho=1 \times 10^{-5} \mathrm{~nm}^{-2}$ \\
Total strain time rate & $\dot{\varepsilon}=1.9 \times 10^{-3} \mathrm{~s}^{-1}$
\end{tabular}

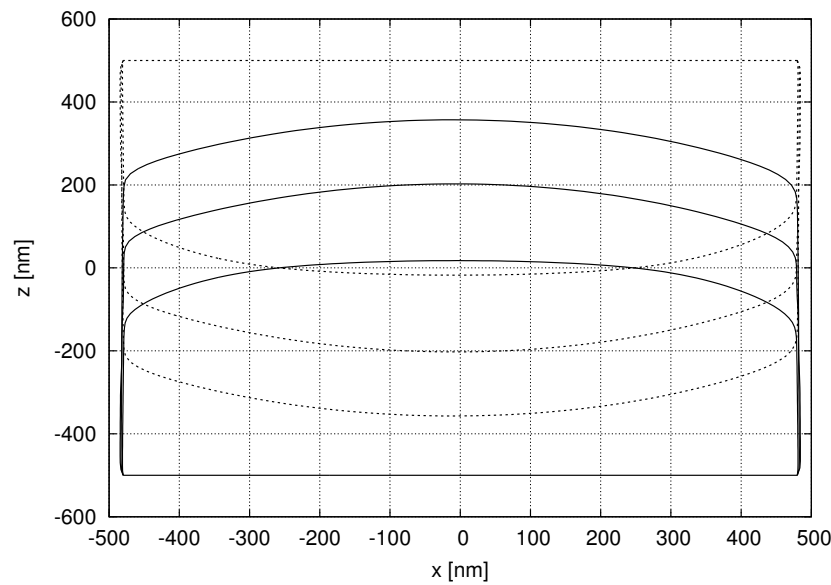

Fig. 2. Dislocations passing each other in the strain control regime. The dislocations evolve from straight line initial states. After overlapping, they continue to glide. Depicted at time levels $t=1.16 \mathrm{~ns}, t=1.56 \mathrm{~ns}$ and $t=1.78 \mathrm{~ns}$

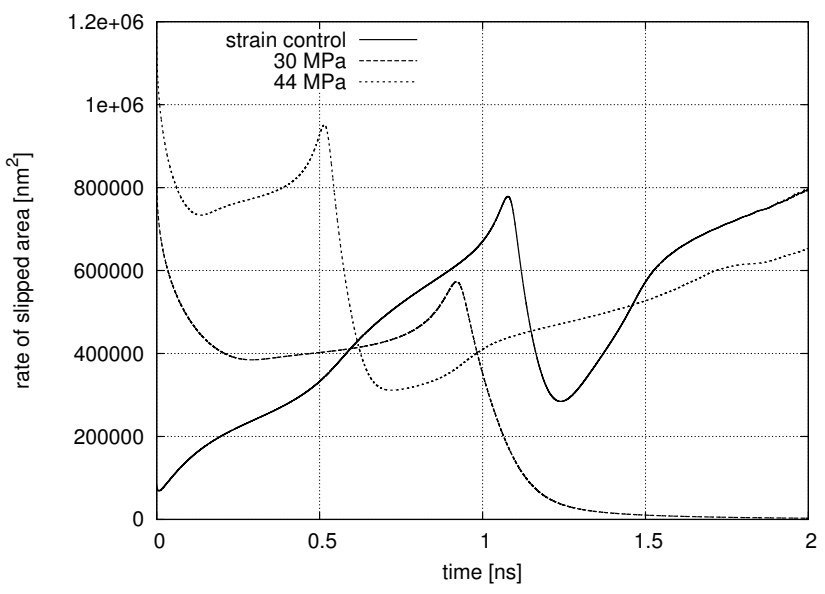

Fig. 3. Comparison of the rate of the swept area for one dislocation. The horizontal axis is the time and the vertical axis is the time derivative of the swept area in $\mathrm{nm}^{2}$. 
In the "stress control regime", the situation is relatively simple, yet computationally complex. We use the approach the interval bisection and perform several simulations with various values of the applied stress. In the simulation data, we can observe and determine several quantities to estimate the passing stress. In this contribution, the quantity of choice is the rate of area swept by the dislocation (see Fig. 3). When the rate of the swept area reaches zero, it indicates that dislocations reached the steady state and formed a dipole. From the performed numerical experiments, we observe that for the stress control regime, the value $\tau_{\text {app }}=30-31 \mathrm{MPa}$ is the maximal stress, where the dislocations still form a dipole and the passing stress is about $\tau_{\text {pass }}=31-32 \mathrm{MPa}$.

In the "strain control regime", such approach typically fails (see the solid curve in Fig. 3), since the total shear strain increases (linearly in our model) in time and dislocations always pass. Instead, one can adapt the method proposed by Mughrabi and Pschenitzka in [14], where the observed quantity is the overall stress $\tau$ :

$$
\tau(u, t)=B v(u, t)-\tau_{\text {app }}(u, t)
$$

for the parameter $u$ belonging to $[0,1]$. To neglect the effect of channel walls, we measure this quantity in the middle of the channel, i.e., for $u=0.5$. The escape stress is then identified with the local maximum of the overall stress $\tau$. In Fig. 4, it can be seen that the passing stress

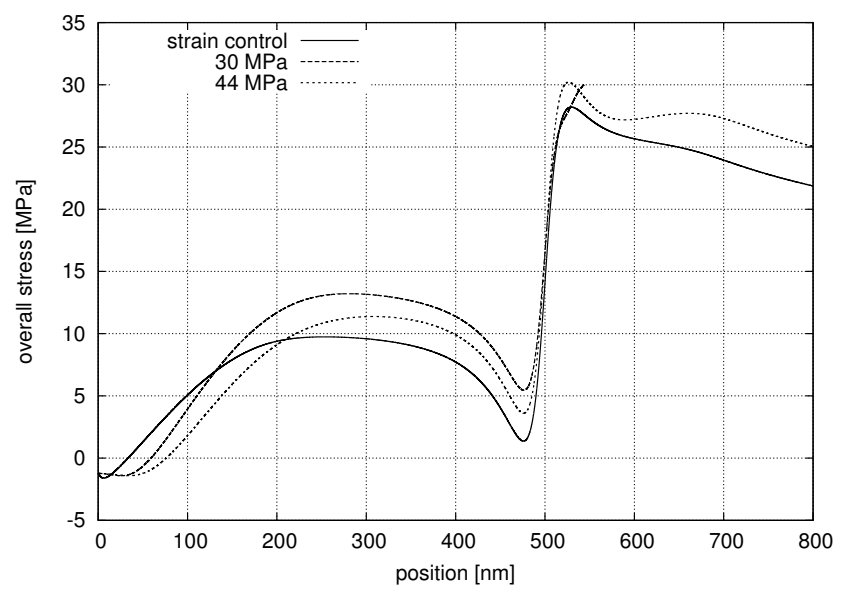

Fig. 4. The overall stress in the middle of the channel depending on the slipped distance of the middle point of the dislocation. The local maximum identified with the escape stress is about $28.2 \mathrm{MPa}$ for the strain control regime and $30.2 \mathrm{MPa}$ for the stress control regime.

is about $\tau_{\text {pass }}=28.2 \mathrm{MPa}$, which is in agreement with the expectation, that the strain control regime provides the lower estimate of the passing stress. This method provides quite reasonable estimate of the passing stress $(30.2 \mathrm{MPa})$ for the stress control regime as well - the curve representing the overall stress for $\tau_{\text {app }}=44 \mathrm{MPa}$ reaches its local maximum of $30.2 \mathrm{MPa}$. One can also notice that the curve representing the overall stress of dipole formation $\left(\tau_{\mathrm{app}}=30 \mathrm{MPa}\right)$ reaches its maximum of $30 \mathrm{MPa}$ in the steady state. We compared our results with the results published by Křišt'an and Kratochvíl in [11] as well, where the estimate of the passing stress for the $h=55 \mathrm{~nm}$ is about $26.7 \mathrm{MPa}$. In good agreement, our implementation provided the value 27.3 MPa.

\section{Conclusion}

The objective of the presented paper was to estimate the passing stress in the PSB channel. We investigated the stress controlled regime where we determined the passing stress by bisection method. The method provided the passing stress $\tau_{\text {pass }} \approx 30-31 \mathrm{MPa}$. Considering the strain controlled regime, the investigation of the overall stress provided the passing stress $\tau_{\text {pass }} \approx 28.2 \mathrm{MPa}$ (note that this value is only approximate - in stress controlled regime, this setting formed a dipole). The results of the numerical experiments confirmed that the stress controlled regime provides the upper estimate, and the strain controlled regime provides the lower estimate of the passing stress. The difference is about $10 \%$. It provides an evidence about a possible inaccuracy hidden in standard DDD simulations.

\section{Acknowledgments}

The research has been partly supported by the project No. P108/12/1463 of the Grant Agency of the Czech Republic.

\section{References}

[1] B. Devincre, Solid State Commun. 93, 875 (1995).

[2] N. Ghoniem, L. Sun, Phys. Rev. B 60, 128 (1999).

[3] A. Vattré, B. Devincre, F. Feyel, R. Gatti, S. Groh, O. Jamond, A. Roos, J. Mech. Phys. Solids 63, 491 (2014).

[4] J. Křišt'an, J. Kratochvíl, V. Minárik, M. Beneš, Modell. Simul. Mater. Sci. Eng. 17, 045009 (2009).

[5] P. Pauš, M. Beneš, J. Kratochvíl, Acta Mater. 61, 7917 (2013).

[6] M. Beneš, J. Kratochvíl, J. Křrišt'an, V. Minárik, P. Pauš, Eur. Phys. J. ST 177, 177 (2009).

[7] P. Pauš, M. Beneš, Kybernetika 45, 591 (2009).

[8] M. Kolář, M. Beneš, D. Ševčovič, J. Kratochvíl, IAENG Int. J. Appl. Math. 45, 198 (2015).

[9] D. Hull, D. Bacon, Introduction to Dislocations, Butterworth-Heinemann, 2011.

[10] M. Peach, J.S. Koehler, Phys. Rev. 80, 436 (1950).

[11] J. Křišt'an, J. Kratochvíl, Philos. Mag. 87, 4593 (2007).

[12] V. Minárik, M. Beneš, J. Kratochvíl, J. Appl. Phys. 107, 061802 (2010).

[13] D. Ševčovič, S. Yazaki, Jpn. J. Ind. Appl. Math. 28, 413 (2011).

[14] H. Mughrabi, F. Pschenitzka, Philos. Mag. 85, 3029 (2005). 